\title{
A New Procedure for Treating a Sebaceous Cyst: Removal of the Cyst Content with a Laser Punch and the Cyst Wall with a Minimal Postponed Excision
}

\author{
HuiLing Wu · ShouJie Wang · LingJiao Wu • \\ ShuSen Zheng
}

Received: 6 November 2008/Accepted: 19 February 2009/Published online: 31 March 2009

(C) The Author(s) 2009. This article is published with open access at Springerlink.com

\begin{abstract}
Three main techniques are used to excise sebaceous cysts: conventional wide excision, minimal excision, and punch biopsy excision. A new method with two steps is proposed. First, a laser is used to make a small hole for removal of the content. Then the cyst wall is removed entirely with a minimal excision about 1 month later. With this method, the cyst is completely removed with only a small scar. It offers a good alternative for eradication of uninfected cysts, especially large cysts or cysts located in areas of thick skin or cosmetic concern.
\end{abstract}

Keywords Laser - Minimal excision - Sebaceous cyst

Sebaceous cysts are benign lesions of the skin, and cosmetic reasons for removal of cysts in an exposed area are often mentioned. A conventional wide excision can remove the cyst completely but with a potentially long scar. The minimal excision technique is similar to the punch technique, which can achieve minimal scarring but carries a risk of recurrence [1-3]. For large cysts or cysts located in areas of thick skin, the cyst wall is more friable or not easy

H. Wu $\cdot$ S. Wang

Department of Plastic Surgery, The First Affiliated Hospital, College of Medicine, Zhejiang University, Hangzhou, China

\section{Wu $(\bowtie)$}

The First Affiliated Hospital, College of Medicine, Zhejiang University College of Medicine, Zhejiang University,

Hangzhou, Zhejiang, China

e-mail: 1020wlj@163.com

\section{S. Zheng}

Department of Hepato-Biliary Surgery, The First Affiliated

Hospital, College of Medicine, Zhejiang University,

Hangzhou, China to express, making complete cyst removal with a better cosmetic appearance more difficult.

Combining the advantages of two methods, we proposed a new method with two steps. First, a laser is used to make a small hole for removal of the cyst content. Then the cyst wall is removed entirely with minimal excision about 1 month later. We have treated 12 sebaceous cysts with this method successfully since 2006 .

\section{Surgical Technique}

After finding the skin conglutination located in the middle part or duct of the anterior cyst wall, we used a carbon dioxide laser to make multiple punctures, creating a hole about 2-4 $\mathrm{mm}$ in diameter (Fig. 1a, b). Partial cystic contents were exuded through the hole by gentle pressure and squeezing (Fig. 1c). If the contents were solid inside, we used a small curette carefully without damaging the wall and washed the remnants with $0.9 \% \mathrm{NaCl}$ solution to ensure that all the contents were removed. Then we sterilized the inside wall of the cyst with povidine-iodine solution. The tiny wound was closed with a single suture or held together with glue. Antibiotic ointment was applied, and the patient was instructed to take antibiotic regularly.

About 1 month later, the cyst wall had become small. By using a no. 15 blade, the skin of the formerly healed wound was incised to a length of approximately $3-5 \mathrm{~mm}$, and the total cyst wall was removed easily (Fig. 1d). The incision closed by natural healing in about 1 week (Fig. 1e).

\section{Results}

The cysts included six in the face, three in the back, one in the neck, and two in other areas. No complications resulted 
Fig. 1 A 0.7-cm cyst developed during 1 year in a 46-year-old man. a View of the cyst. b A hole about $2 \mathrm{~mm}$ in diameter created by multiple punctures of a carbon dioxide laser. c Partial cystic contents exuded through the hole by gentle pressure and squeezing. d Reduced cyst 36 days after the laser treatment. The total cystal wall is easy to remove completely under direct vision. e The resulting scar is not noticeable 1 week after removal of the cystal wall
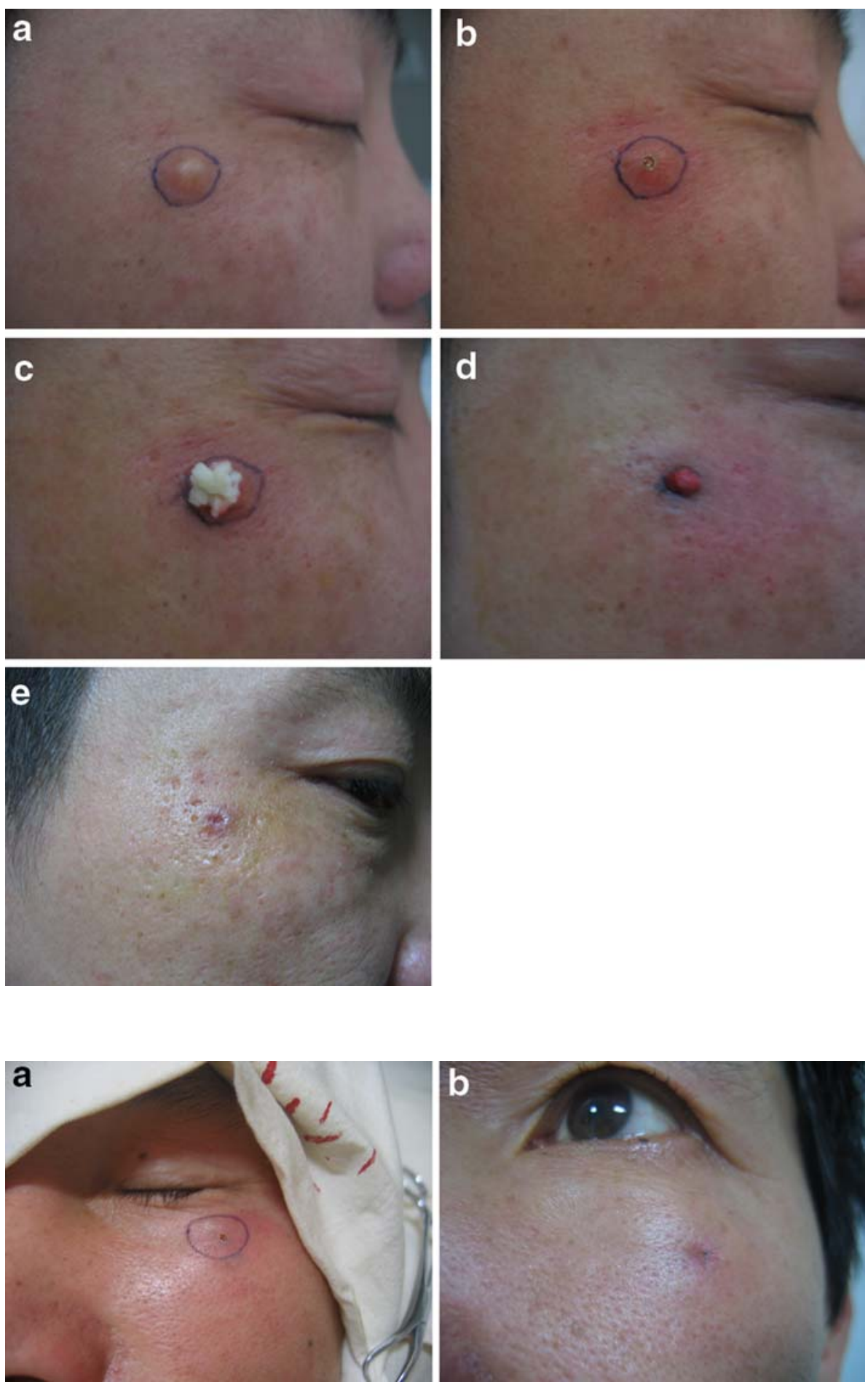

Fig. 2 A 1.5-cm irregular cyst in a 53-year-old man. a View of the cyst with a hole created by carbon dioxide laser. b View 4 days after the total cystal wall removal for any of these cysts. The average cyst diameter was $1.36 \mathrm{~cm}$, and the length of the resulting scar was only about one-third the diameter of the initial cyst. For some small lesions on the face, the scar was inconspicuous (Figs. 1e and $2 \mathrm{a}, \mathrm{b})$. Telephone interviews were conducted for all the patients after a follow-up period of 3-24 months with no recurrence.

\section{Discussion}

Most clinicians suggest that if cyst removal is desired or indicated, every effort should be made to remove the entire cyst lining to prevent recurrence of the sebaceous cyst [1]. But with excision of a large cyst on the face, neck, or back, it is difficult to achieve an aesthetically pleasing closure 
[4]. Thin-walled cysts tend to break and may need to be removed in pieces [5], with the risk of recurrence when the minimal excision technique is used.

The method we propose has some advantages. The laser is easy to manipulate, and the laser hole punch results in little bleeding, giving a clear field of vision for the operating area and no infection. After expulsion of the cyst contents, the capsule is gradually loosened and crumples to a smaller capsule with some fluid inside. Its complete removal is easy in the delayed operation, which allows for dissection and excision under direct vision, providing for confident and complete removal of the cyst wall while minimizing scar length. The length of the resulting scar is only about one-third that of the initial cyst diameter, and for some small lesions on the face, the scar is inconspicuous.

\section{Conclusion}

The described surgical procedure produces no recurrence, with favorable cosmetic results. It can be a good alternative for eradication of uninfected cysts, especially large cysts or cysts located in areas of thick skin or cosmetic concern.
Open Access This article is distributed under the terms of the Creative Commons Attribution Noncommercial License which permits any noncommercial use, distribution, and reproduction in any medium, provided the original author(s) and source are credited.

\section{References}

1. Moore RB, Fagan EB, Hulkower S, Skolnik DC, O'Sullivan G (2007) Clinical inquiries: what's the best treatment for sebaceous cysts? J Fam Pract 56:315

2. Avakoff JC (1989) Microincision for removing sebaceous cysts. Plast Reconstr Surg 84:173

3. Nakamura M (2001) Treating a sebaceous cyst: an incisional technique. Aesth Plast Surg 25:52

4. Rao K, Tehrani H (2006) Excision of epidermoid cysts with a minimal linear incision. Dermatol Online J 12:21

5. Zuber TJ (2002) Minimal excision technique for epidermoid (sebaceous) cysts. Am Fam Physician 65:1409, 1417, 1420 